\section{ORIGINAL} RESEARCH

T. Niwa

N. Aida

A. Shishikura

K. Fujita

T. Inoue

\title{
Susceptibility-Weighted Imaging Findings of Cortical Laminar Necrosis in Pediatric Patients
}

BACKGROUND AND PURPOSE: MR susceptibility-weighted imaging (SWI) is a highly sensitive technique for detection of hemorrhage, but its utility in the evaluation of children with laminar necrosis is not yet known. We assessed whether cortical laminar necrosis in pediatric patients contains hemorrhage on SWI.

MATERIALS AND METHODS: "Cortical laminar necrosis" was defined as a hyperintense cortical lesion on T1-weighted imaging in the subacute or chronic phase of brain damage in some foci involving the cerebral cortex and white matter such as hypoxic-ischemic incidents and encephalopathy. Medical records, CT, and MR images were retrospectively analyzed. Fifteen patients ( 7 boys, 8 girls; age range, 0-13 years) were included. The areas of signal-intensity loss on SWI that were considered to be hemorrhage were correlated with the laminar necrosis. CT was assessed to correlate with the presence of calcification at the location of the signal-intensity loss on SWI. To assess appearance or signal-intensity changes of hemorrhage in the laminar necrosis, follow-up SWI was performed.

RESULTS: The causes of laminar necrosis included infarction in 4 patients, ischemic changes from Moyamoya disease in 2, meningoencephalitis in 2, hypoxic-ischemic encephalopathy in 2, shaken baby syndrome in 1, encephalopathy from severe infection in 1, status epilepticus in 1, citrullinemia in 1, and brain injury with posterior reversible encephalopathy syndrome in 1. T1-weighted imaging showed focal laminar necrosis in 8, multifocal laminar necrosis in 2, and diffuse laminar necrosis in 5. SWI findings correlated with laminar necrosis included the following: no hemorrhage in 13 patients (80.0\%), dotted hemorrhage in 2 (13.3\%), and laminar hemorrhage in 1 (6.7\%). Follow-up SWI performed in 6 patients showed no additional hemorrhage.

CONCLUSION: Most areas of cortical laminar necrosis in pediatric patients showed no hemorrhage on SWI.

C ortical laminar necrosis is caused by cerebral energy depletion, resulting in necrosis of particular cortical laminae. ${ }^{1}$ On T1-weighted MR images, hyperintense lesions are seen in the cerebral cortex during the subacute or chronic stage of brain damage. Cortical laminar necrosis has been described in different conditions. In children, cortical laminar necrosis can be caused by various diseases, including hypoxic-ischemic encephalopathy, infarction, status epilepticus, Moyamoya disease, metabolic disorders, and intoxication. ${ }^{1-5}$ Because varying brain damage leads to laminar necrosis, it is important to understand the underlying pathology and clinical outcome with imaging findings.

MR susceptibility-weighted imaging (SWI) was designed to be a high-spatial-resolution 3D fast low-angle shot MR imaging technique; it is extremely sensitive to susceptibility changes and can be performed by using conventional MR imaging units. ${ }^{6}$ This sequence was originally designed for MR venography and involves the use of the paramagnetic property of intravenous deoxyhemoglobin. Recently, SWI has been reported to be very sensitive for the detection of extravascular blood products, and several investigators have shown that it has higher sensitivity to detect hemorrhage than conventional MR imaging. ${ }^{7,8}$ Detection of hemorrhage is important in the

Received February 25, 2008; accepted after revision April 23.

From the Department of Radiology (T.N., N.A., A.S., K.F.), Kanagawa Children's Medical Center, Yokohama, Japan; and Department of Radiology (T.I.), Yokohama City University School of Medicine, Yokohama, Japan.

Please address correspondence to Tetsu Niwa, MD, Department of Radiology, Kanagawa Children's Medical Center, 2-138-4 Mutsukawa, Minami-ku, Yokohama, 232-8555, Japan; e-mail: tniwa@kcmc.jp

DOI 10.3174/ajnr.A1184 diagnosis and management of a variety of intracranial diseases. Treatments such as thrombolysis and imaging follow-up interval might be affected by the presence of hemorrhage. To the best of our knowledge, the presence of hemorrhage in cortical laminar necrosis has not been wellestablished in pediatric patients. The purpose of this study was to identify, by using SWI, whether cortical laminar necrosis contains hemorrhage in these patients.

\section{Materials and Methods}

\section{Patients}

"Cortical laminar necrosis" was defined as hyperintense cortical lesions on T1-weighted imaging found during the subacute or chronic phase of brain damage.

One patient was excluded because of severe MR imaging artifact from a shunt device, and 15 patients ( 7 boys, 8 girls; age range, 1 week to 13 years; mean age, 4.3 years) were included. Medical records, CT images, and MR images were retrospectively reviewed. Our institutional review board did not require its approval or informed consent for the retrospective evaluation of patients' records and images.

\section{MR Imaging}

All MR imaging was performed with a $1.5 \mathrm{~T}$ superconducting MR imaging system (Avanto; Siemens, Erlangen, Germany) with a 33 $\mathrm{mT} / \mathrm{m}$ maximal gradient capability and a maximal slew rate of 125 $\mathrm{mT} / \mathrm{m}$ per millisecond. Scanning was performed with a combination of high-resolution surface coils for neonates and infants and a headcoil for older children. Sedating agents were used when necessary. The routine MR imaging protocol included transverse T1-weighted spinecho; transverse and sagittal or coronal T2-weighted fast spin-echo; and transverse diffusion-weighted imaging. Imaging parameters var- 


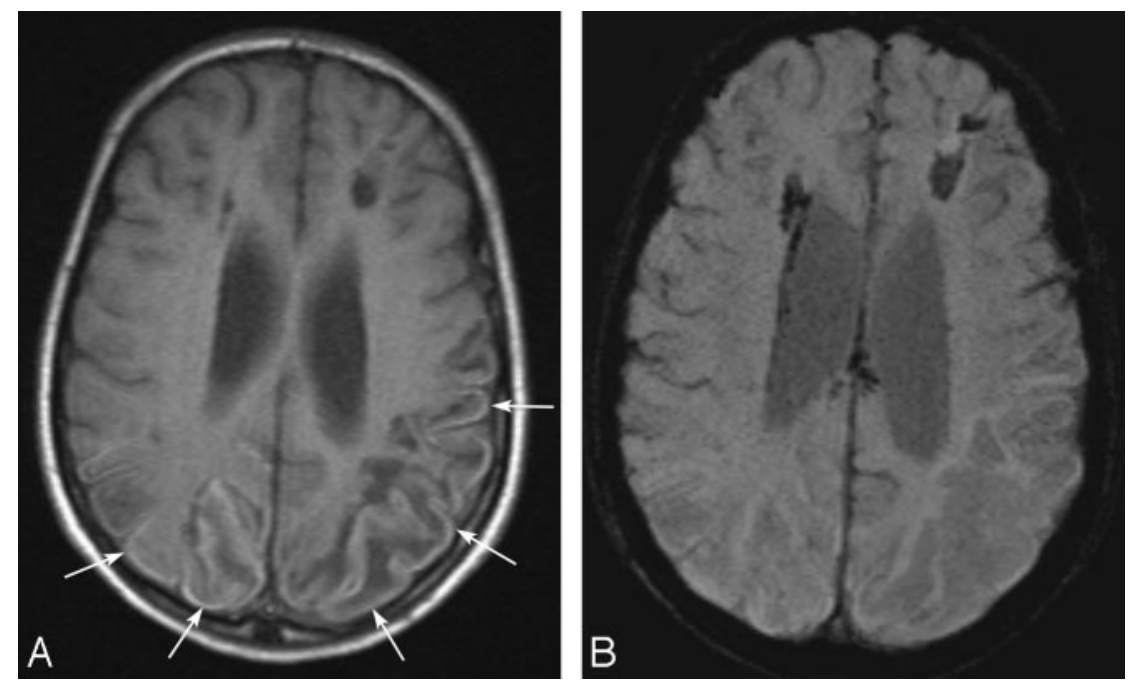

Fig 1. A 13-year-old boy with ischemic changes of Moyamoya disease. A, Transverse T1-weighted spin-echo image shows curvilinear hyperintense cortical lesions in the bilateral parietal lobes (arrows). B, Corresponding transverse susceptibility-weighted image shows no signal-intensity loss indicating hemorrhage in the cortical lesions.

ied depending on the patient's development ${ }^{9}$ and head size. The actual sequences were the following: T1-weighted spin-echo imaging (TR, 485-523 msec; TE, 9.3-11 ms; 1 signal acquired), T2-weighted fast spin-echo imaging (TR/TE, 4530-5360/110-140; 1 signal acquired), and diffusion-weighted imaging (TR/TE, 5000/94 ms; 2 signals acquired; generalized autocalibrating partially parallel acquisition [GRAPPA], 2). The FOV was set to $141-167 \times 150-200 \mathrm{~mm}$, the matrix size was set to $119-144 \times 192-256$, and the section thickness was set to $5 \mathrm{~mm}$ with a $1-\mathrm{mm}$ intersection gap.

SWI consisted of a strongly susceptibility-weighted low-bandwidth $(80 \mathrm{~Hz} /$ pixel $) 3 \mathrm{D}$ fast low-angle shot sequence, which was firstorder flow-compensated in all 3 orthogonal directions. The parameters for SWI were the following: TE/TR, 69/60 ms for neonates, 59/50 $\mathrm{ms}$ for infants, and 49/40 ms for children older than 12 months; flip angle, $20^{\circ}$; section thickness, 1.3-2-mm; FOV, $150 \times 200-250 \mathrm{~mm}$; matrix size, $130-150 \times 256$; GRAPPA, 2 . With the SWI sequence, most cerebral hemispheres were imaged within an acquisition time of approximately $4-5$ minutes. SWI was created by using the magnitude and phase images. The normalized phase mask was multiplied 4 times against the original magnitude image. ${ }^{7,10}$ Finally, a minimum intensity projection was performed over 2 or 3 sections to display the processed data by using contiguous approximately 4 -mm-thick sections in the transverse plane.

Additional planes and sequences, such as fluid-attenuated inversion recovery and contrast-enhanced T1-weighted images, were obtained depending on the disease.

\section{Data Analysis}

Cortical laminar necrosis was defined as a hyperintense cortical lesion on T1-weighted imaging in the subacute or chronic phase of brain damage in some foci involving the cerebral cortex and white matter. MR images were evaluated by 2 experienced radiologists to determine the presence or absence of signal-intensity loss in the cortical laminar necrosis, on the workstation (Synapse; Fujifilm Medical Systems, Tokyo, Japan), which was routinely used to view images. The evaluations were the result of the consensus of the 2 radiologists. If a signalintensity loss area was noted on SWI, the location of the lesion was compared with that of the hyperintense cortical lesion on the T1weighted image. Hemorrhagic lesions were defined as signal-intensi- ty-loss foci on SWI that were not compatible with vascular, bone, or artifactual structures correlated with conventional images. Because SWI is sensitive for detecting extravascular blood products, as well as other substances such as iron, some forms of calcification, and air, ${ }^{11}$ CT was assessed to correlate with the presence of calcification at the location of the signal-intensity loss detected on SWI.

To assess the appearance of hemorrhage or signal-intensity changes of hemorrhage in the laminar necrosis, we performed follow-up SWI. For patients who had serial SWI examinations, the appearance of hemorrhage or signal-intensity changes of hemorrhage in the laminar necrosis on SWI were also assessed. For patients with hemorrhage in the laminar necrosis detected on SWI, follow-up CT and/or MR images were reviewed for changes in the hemorrhagic area.

\section{Results}

The causes of laminar necrosis included the following: infarction in 4 patients, Moyamoya disease in 2, hypoxic-ischemic injury in 2, meningoencephalitis in 2 , shaken baby syndrome in 1, encephalopathy from severe infection in 1, status epilepticus in 1 , citrullinemia in 1 , and cerebral injury from posterior reversible encephalopathy syndrome secondary to tacrolimus in 1. All MR images were of sufficient quality to evaluate laminar necrosis on T1-weighted imaging and signal-intensity loss on SWI. Duration between symptom onset and initial SWI ranged from approximately 2 weeks to 490 days. In 3 patients, the symptom onset was uncertain.

T1-weighted images showed focal laminar necrosis in 8 patients, multifocal laminar necrosis in 2, and diffuse laminar necrosis in 5. Laminar necrosis was identified in 1 cerebral hemisphere in 5 patients and in both cerebral hemispheres in 10 patients. On SWI, most areas of laminar necrosis did not show hemorrhage (Fig. 1). SWI findings correlated with laminar necrosis included the following: no hemorrhage in 12 patients $(80.0 \%)$, dotted hemorrhage in $2(13.3 \%)$, and focal laminar hemorrhage in 1 (6.7\%) (Fig 2). The underlying disease of the 3 patients with hemorrhage in the laminar necrosis included 1 case of meningoencephalitis in a 13-year-old boy and 1 case of infarction in a 12-month-old girl among those 

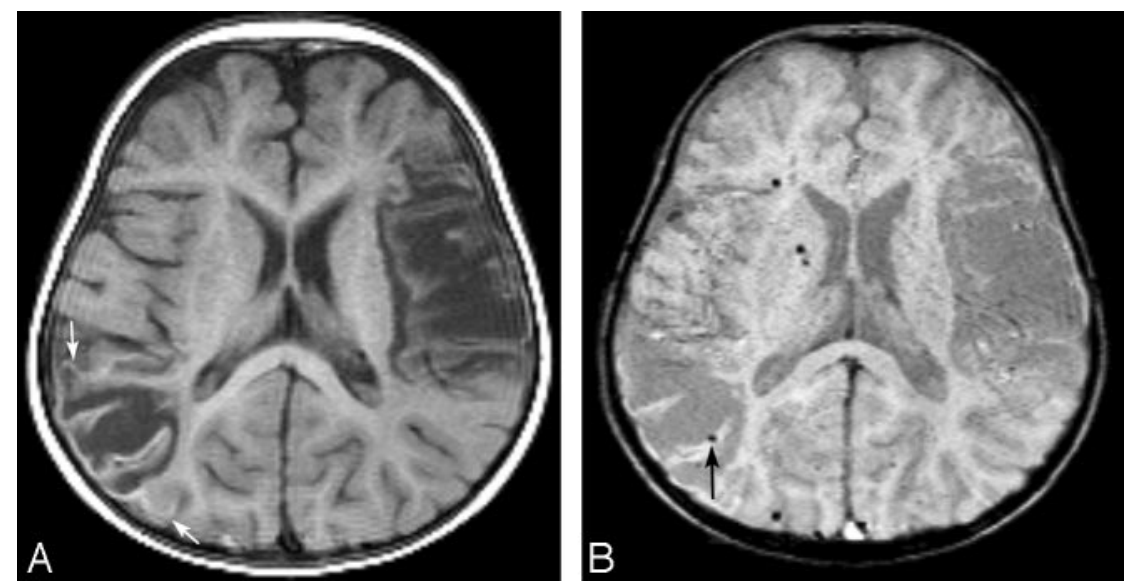

Fig 2. A 12-month-old girl with chronic infarction in bilateral middle cerebral artery territories. This patient had prior heart surgery. A, Transverse $T 1$-weighted image shows a curvilinear hyperintense cortical lesion (arrows). B, Corresponding transverse susceptibility-weighted image shows dotted signal-intensity loss (arrow), which indicates hemorrhage, in the cortical lesions. Although we consider the signal-intensity loss on the laminar necrosis as hemorrhage because of discontinuity, it is difficult to strictly distinguish it from part of the cortical vein. Note that several small foci of dotted hemorrhage are also found in other parts of the brain parenchyma.
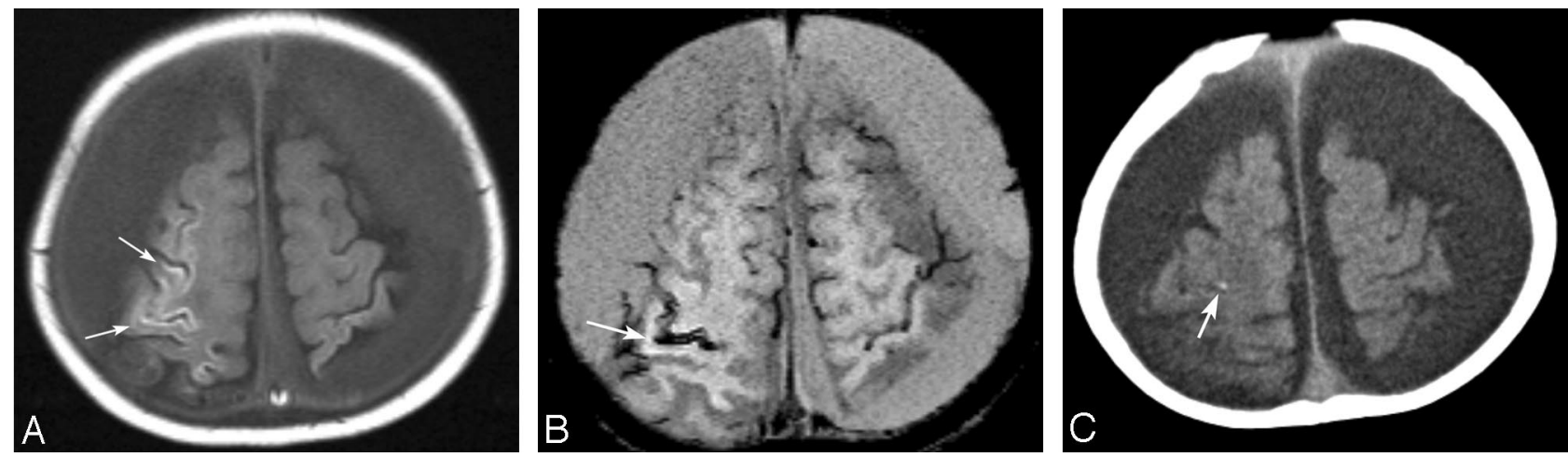

Fig 3. An 8-month-old boy with shaken baby syndrome. A, Transverse T1-weighted image shows a curvilinear hyperintense cortical lesion in the damaged area (arrows). $B$, Susceptibility-weighted image shows laminar signal-intensity loss, which indicates hemorrhage, along with some cortical lesions (arrow). $C$, CT scan shows dotted calcification (arrow) in part of the area of laminar hemorrhage on SWI.

with dotted hemorrhage, and shaken baby syndrome in association with focal laminar hemorrhage in 1 case of an 8-month-old boy. The patient with shaken baby syndrome showed dotted calcification on CT in part of the laminar hemorrhage (Fig 3); the 2 patients with dotted hemorrhage showed no calcification on CT.

Serial SWI was performed in 6 patients. The time from the initial examination to the follow-up examination ranged from 49 to 483 days. In all patients who underwent serial SWI, cortical hyperintensity remained on the T1-weighted images. Five of the 6 patients showed no hemorrhage in the laminar necrosis on both the initial and follow-up SWI. One patient with brain damage who had meningoencephalitis, who showed dotted hemorrhage on the initial SWI, demonstrated reduction of the signal-intensity loss on follow-up SWI. In the follow-up examinations that occurred from 57 to 438 days from the initial SWI examination, no patients showed subsequent hematoma development in the area of hemorrhage in the laminar necrosis.

\section{Discussion}

Most areas of laminar necrosis, which occurred as a result of various cerebral disorders in pediatric patients, did not show hemorrhage on SWI. Several investigators surveyed the rela- tionship between hemorrhage and laminar necrosis in adult patients with chronic cerebral infarction by using $\mathrm{T} 2{ }^{*}$ weighted gradient-echo imaging; they reported that laminar necrosis did not represent hemorrhage in cortical curvilinear hyperintense lesions on T1-weighted imaging. ${ }^{6,12}$ MR imaging-pathologic correlation studies in autopsied patients with chronic cerebral infarction demonstrated that cortical hyperintense lesions on T1-weighted images corresponded to necrotic debris throughout the entire cortex without hemorrhage or calcification. ${ }^{6,13}$ Our results by using SWI generally support the results of previous studies.

In the present study, small foci of hemorrhage were detected in 1 patient with meningoencephalitis, 1 with subacute infarction, and 1 with shaken baby syndrome. The 1 patient with meningoencephalitis with dotted hemorrhage in the laminar necrosis also had a mass effect from an empyema that had been drained. These factors may have caused the dotted hemorrhage. A T1 and T2 prolonged area, which might reflect brain damage or gliosis, was seen in the subcortical region near the dotted hemorrhage. The patient with subacute infarction with dotted hemorrhage in the laminar necrosis showed several foci of dotted hemorrhage in the brain parenchyma, which included areas other than the area of the infarction. This patient had undergone heart surgery, and the infarction prob- 
ably occurred several days after the surgery. The dotted hemorrhage in the laminar necrosis in this patient may have had no relation to the infarction, and 1 focus of hemosiderin might have occurred in the area of laminar necrosis incidentally.

Recent studies have suggested that the most severe clinical and histologic manifestations of shaken baby syndrome are related to hypoxic-ischemic damage, whereas widespread axonal damage appears to be less common than once thought. ${ }^{14-16}$ However, this topic remains controversial. ${ }^{17}$ In the present study, dotted calcification was seen in part of the laminar hemorrhage in a patient with shaken baby syndrome, and the remaining laminar signal-intensity loss in the area of laminar necrosis should be considered hemorrhage. Complicated mechanisms in shaken baby syndrome may contribute to laminar hemorrhage in the laminar necrosis detected in the present study. Cortical laminar necrosis in shaken baby syndrome may have a different neuropathologic status than that resulting from ischemia.

Recent studies have shown that SWI is superior for detecting hemorrhage compared with gradient-echo-sequence images or CT in the acute and subacute phases. ${ }^{7,8}$ Most blood products, including deoxyhemoglobin, methemoglobin, and hemosiderin, are paramagnetic and thus make it possible to exploit susceptibility effects visible on SWI as signal-intensity loss. To the best of our knowledge, detection of hemorrhage in laminar necrosis with SWI has not been previously reported. Laminar necrosis in adult patients in the subacute or chronic phase of infarction has been considered not to represent hemorrhage. ${ }^{6}$ However, hemorrhagic foci were occasionally identified. ${ }^{18,19}$ Laminar necrosis has been identified in the varying brain damage in children, and underlying pathology may not be the same status. In our series, only small foci of hemorrhage were identified in a few patients. Although previous studies showed no hemorrhage in the laminar necrosis with gradientecho-sequence images, small foci of hemorrhage may be present in a part of the laminar necrosis on SWI in some patients such as those having brain impaction or shaken baby syndrome due to its higher sensitivity for hemorrhage or underlying complicated pathology.

Cortical laminar necrosis may remain for $1.5-2$ years. ${ }^{12,20}$ Kashihara et $\mathrm{al}^{21}$ reported that cortical laminar necrosis was observed in a patient with central nervous system lupus erythematosus for 5 years. Serial imaging studies of laminar necrosis by using $\mathrm{CT}$ and $\mathrm{T} 2^{*}$-weighted images showed no hemorrhage. ${ }^{12,20}$ In the present study, no increase of hemorrhage or subsequent hematoma was found in the laminar necrosis on follow-up examination. Thus, hemorrhage in the laminar necrosis, if present, may involve a limited area and should not be considered as related to subsequent hematoma.

The present study had several limitations. First, hemorrhage was defined as a discontinuous signal-intensity loss, but small foci of signal-intensity loss on SWI in the cortical portion are difficult to distinguish from part of the cortical vein owing to blooming effects. ${ }^{7}$ Second, signal-intensity loss was considered to be hemorrhage because calcification was not seen with CT. However, other materials with a strong susceptibility effect such as nonhemorrhagic iron deposition could not be ruled out. Third, the MR imaging parameters used for SWI differed depending on age, because in clinical practice, it was necessary to assess the presence of hemorrhage and the venous system. Fourth, because this was a retrospective study, the timing of the $\mathrm{CT}$ and MR imaging scans varied depending on the patient's status.

\section{Conclusions}

Most areas of cortical laminar necrosis in pediatric patients showed no hemorrhage on SWI. Hemorrhage in cortical laminar necrosis appears to be uncommon in these patients.

\section{References}

1. van der Knaap MS, Smit LS, Nauta JJ, et al. Cortical laminar abnormalities: occurrence and clinical significance. Neuropediatrics 1993;24:143-48

2. Riudavets MA, Aronica-Pollak P, Troncoso JC. Pseudolaminar necrosis in cyanide intoxication: a neuropathology case report. Am J Forensic Med Pathol 2005;26:189-91

3. Kinoshita T, Takahashi S, Ishii K, et al. Reye's syndrome with cortical laminar necrosis: MRI. Neuroradiology 1996;38:269-72

4. Takeda K, Takahashi S, Higano S, et al. Cortical laminar necrosis in a patient with moyamoya disease associated with Down syndrome: MR imaging findings. Radiat Med 1997; 15:59-63

5. Bargallo N, Burrel M, Berenguer J, et al. Cortical laminar necrosis caused by immunosuppressive therapy and chemotherapy. AJNR Am J Neuroradiol 2000;21:479-84

6. Kinoshita T, Ogawa T, Yoshida Y, et al. Curvilinear T1 hyperintense lesions representing cortical necrosis after cerebral infarction. Neuroradiology 2005; $47: 647-51$

7. Tong KA, Ashwal S, Holshouser BA, et al. Hemorrhagic shearing lesions in children and adolescents with posttraumatic diffuse axonal injury: improved detection and initial results. Radiology 2003;227:332-39

8. Wycliffe ND, Choe J, Holshouser B, et al. Reliability in detection of hemorrhage in acute stroke by a new three-dimensional gradient recalled echo susceptibility-weighted imaging technique compared to computed tomography: a retrospective study. J Magn Reson Imaging 2004;20:372-77

9. Barkovich AJ, Kjos BO, Jackson DE Jr, et al. Normal maturation of the neonatal and infant brain: MR imaging at 1.5 T. Radiology 1988;166:173-80

10. Reichenbach JR, Venkatesan R, Schillinger DJ, et al. Small vessels in the human brain: MR venography with deoxyhemoglobin as an intrinsic contrast agent. Radiology 1997;204:272-77

11. Tong KA, Ashwal S, Obenaus A, et al. Susceptibility-weighted MR imaging: a review of clinical applications in children. AJNR Am J Neuroradiol 2008;29:9-17

12. Komiyama M, Nakajima H, Nishikawa M, et al. Serial MR observation of cortical laminar necrosis caused by brain infarction. Neuroradiology 1998;40:771-77

13. Boyko OB, Burger PC, Shelburne JD, et al. Non-heme mechanisms for T1 shortening: pathologic, CT, and MR elucidation. AJNR Am J Neuroradiol 1992;13:1439-45

14. Geddes JF, Hackshaw AK, Vowles GH, et al. Neuropathology of inflicted head injury in children. I. Patterns of brain damage. Brain 2001;124:1290-98

15. Geddes JF, Vowles GH, Hackshaw AK, et al. Neuropathology of inflicted head injury in children. II. Microscopic brain injury in infants. Brain 2001;124:1299-306

16. Parizel PM, Ceulemans B, Laridon A, et al. Cortical hypoxic-ischemic brain damage in shaken-baby (shaken impact) syndrome: value of diffusionweighted MRI. Pediatr Radiol 2003;33:868-71

17. McKinney AM, Thompson LR, Truwit CL, et al. Unilateral hypoxic-ischemic injury in young children from abusive head trauma, lacking craniocervical vascular dissection or cord injury. Pediatr Radiol 2008;38:164-74

18. Takahashi S, Higano S, Ishii K, et al. Hypoxic brain damage: cortical laminar necrosis and delayed changes in white matter at sequential MR imaging. $R a-$ diology 1993;189:449-56

19. Manz HJ, Colon AR. Neuropathology, pathogenesis, and neuropsychiatric sequelae of Reye syndrome. J Neurol Sci 1982;53:377-95

20. Siskas N, Lefkopoulos A, Ioannidis I, et al. Cortical laminar necrosis in brain infarcts: serial MRI. Neuroradiology 2003;45:283-88

21. Kashihara K, Fukase S, Kohira I, et al. Laminar cortical necrosis in central nervous system lupus: sequential changes in MR images. Clin Neurol Neurosurg 1999;101:145-47 